\title{
Improved Collision Cryptanalysis of Authenticated Cipher MORUS
}

\author{
Tairong Shi ${ }^{*}$, Jie Guan, Junzhi Li and Pei Zhang \\ Information Science and Technology Institute, Zhengzhou, Henan, China \\ ${ }^{*}$ Corresponding author
}

\begin{abstract}
MORUS is an authenticated stream cipher designed by Wu et al. and submitted for the third-round of the CAESAR competition. The collision properties of MORUS-640-128 are studied. We propose the necessary conditions for an internal state collision after two-step update, i.e., the Hamming weight of the input difference is at least 5 and the difference is distributed in at least three 32-bit words, which provide the theoretical support for MORUS's resistance against collision attack.
\end{abstract}

Keywords-CAESAR; MORUS; collision cryptanalysis; partition method

\section{INTRODUCTION}

Authenticated encryption algorithm [1] combines encryption with authentication, providing both confidentiality and authenticity simultaneously and has been widely used in the security protocols such as SSL/TLS [2], etc. In order to accelerate the development of authentication encryption, CAESAR competition [3] merges. CAESAR is a large-scale cryptographic competition supported by the US National Institute of Standards and Technology (NIST) like AES [4], eSTREAM [5]. With the advance of the competition, authenticated cipher receives more attentions and develops rapidly. The third-round candidates own excellent performance on security and efficiency which are competitive and attract many researchers [6], [7], [8].

MORUS [9] is a notable family of authenticated stream ciphers designed by $\mathrm{H}$. Wu and $\mathrm{T}$. Huang, which has been selected as a third-round candidate of CAESAR competition. By adopting the idea of block cipher, MORUS uses 5 registers of equal length in its internal state and update each state successively. Three MORUS versions: MORUS-640-128, MORUS-1280-128, and MORUS-1280-256 are recommended with different internal state and key sizes. Mileva et al. [10] have proposed a distinguisher attack on MORUS in non-reuse setting. And Zhang et al. [11] researched on the confusion and diffusion properties of the initialization of MORUS. The research on MORUS is relatively limited currently.

Collision attack [12], [13] is a typical method used in attacking the message authentication. The basic technique is to inject a message difference at certain encryption (or processing the associated date) step and cancel it at a later step.

In this paper, we evaluate the security of authentication by theory deduction. Observations reveal that 32-bit word are not confused completely with internal state. Based on the feature that the 32-bit words in the state element are independent of each other, we utilize the partition method and propose the necessary conditions for an internal state collision after twostep update. There results imply that MORUS resists against collision attack in theory.

This paper is organized as follows. In Section 2, the description of MORUS is provided. In Section 3, the distribution of word difference is given. Section 4 discusses the lower bound of difference weight for collision. Section 5 concludes the paper.

\section{DESCRIPTION OF MORUS}

MORUS operates four phases: initialization, processing of the authenticated data, encryption, and finalization. In this paper we only focus on the processing of associated data and encryption and study the security of MORUS-640-128. MORUS takes a 128-bit key, a 128-bit nonce, and a tag length less than or equal to 128. More details of MORUS-640-128 can be found in [9]. All the "MORUS" in the rest of this paper refers to "MORUS-640-128".

\section{A. Notations}

$S^{i}$ : State at the beginning of $i$-th step, where $i \geq 0$;

$S_{k}^{i}$ : State at the beginning of $k$-th round at $i$-th step, where $S_{k}^{i}=\left(S_{k, 0}^{i}, S_{k, 1}^{i}, S_{k, 2}^{i}, S_{k, 3}^{i}, S_{k, 4}^{i}\right), 0 \leq k \leq 4$;

$S_{k, j}^{i}$ : The $j$-th element of the state $S_{k}^{i} . S_{k, j}^{i}=\left(S_{k, j, 1}^{i}, S_{k, j, 2}^{i}\right.$, $\left.S_{k, j, 3}^{i}, S_{k, j, 4}^{i}\right), 0 \leq j \leq 4$;

$S_{k, j, l}^{i}$ : l-th 32-bit word of the $S_{k, j}^{i}$, where $1 \leq l \leq 4$, $0 \leq j \leq 4$;

\& : Bit-wise AND;

$<<<$ : Rotation to the left;

>>> : Rotation to the right;

$\lceil x\rceil$ : The smallest integer not less than $x$;

$\operatorname{Rotl}(x, n)$ : Divide the 128-bit block $x$ into four 32-bit words and rotate each word left by $n$ bits,

$\operatorname{Rotl}^{-1}(x, n)$ : Analogous to $\operatorname{Rotl}(x, n)$, divide the 128-bit $x$ into four 32-bit words and rotate each word right by $n$ bits; 
$1^{n}\left(0^{n}\right): n$ bits of '1's ('0's);

$\mathrm{W}(\alpha)$ : The hamming weight of $\alpha$, namely, the number of 1 in $\alpha$ 's binary representation;

$\operatorname{WB}\left(\Delta m_{t}\right)$ : The number of nonzero word in $\Delta m_{t} \cdot \Delta m_{t}$ denotes the message difference, where $\alpha_{i}(1 \leq i \leq 4)$ represents a 32-bit word difference.

\section{B. The State Update Function of MOURS}

MORUS uses five 128-bit registers in its internal state. This function consists of five rounds with similar operations that update the state $S$.

$S^{i+1}=$ StateUpdate $\left(S^{i}, m_{i}\right)$ is given as follows:

Round 1:

$S_{1,0}^{i}=\operatorname{Rotl}\left(S_{0,0}^{i} \oplus\left(S_{0,1}^{i} \& S_{0,2}^{i}\right) \oplus S_{0,3}^{i}, b_{0}\right) ;$

$S_{1,3}^{i}=S_{0,3}^{i} \ll<w_{0}$;

$S_{1,1}^{i}=S_{0,1}^{i}$;

$S_{1,2}^{i}=S_{0,2}^{i}$;

$S_{1,4}^{i}=S_{0,4}^{i}$;

Round 2 to 5:

For $k=1$ to 4 ,

$$
\begin{aligned}
& S_{(k+1) \bmod 5, k}^{i}=\operatorname{Rotl}\left(S_{k, k}^{i} \oplus\left(S_{k,(k+1) \bmod 5}^{i} \& S_{k,(k+2) \bmod 5}^{i}\right) \oplus\right. \\
& \left.S_{k,(k+3) \bmod 5}^{i} \oplus m_{i}, b_{k}\right) ; \\
& S_{(k+1) \bmod 5,(k+3) \bmod 5}^{i}=S_{k,(k+3) \bmod 5}^{i}<<<W_{k} ; \\
& S_{(k+1) \bmod 5,(k+1) \bmod 5}^{i}=S_{k,(k+1) \bmod 5}^{i} ; \\
& S_{(k+1) \bmod 5,(k+2) \bmod 5}^{i}=S_{k,(k+2) \bmod 5}^{i} ; \\
& S_{(k+1) \bmod 5,(k+4) \bmod 5}^{i}=S_{k,(k+4) \bmod 5}^{i} .
\end{aligned}
$$

Generate $S^{i+1}$ : For $k=0$ to $4: S_{k}^{i+1}=S_{k}^{i}$.

The rotation constants $b_{i}, w_{i}, i=0, \cdots, 4$ for each round are defined in [10].

\section{Processing the Associated Data and Encryption}

The Processing the associated data $A D$ of MORUS can be described as follows. adlen means bit length of the associated data, where $u=\lceil$ adlen $/ 128\rceil$.

For $i=0$ to $u-1: S^{i}=\operatorname{StateUpdate}\left(S^{i}, A D_{i}^{128}\right)$.

The encryption is described as follows. Let msglen represents the length of plaintext and $v=\lceil m s g l e n / 128\rceil$.

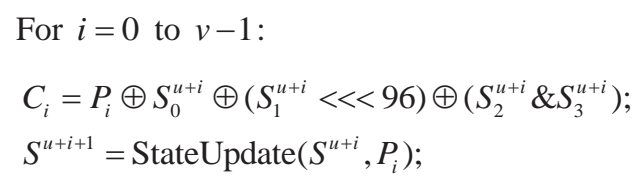

\section{The Distribution OF WORD-ORIENTED DIFFERENCE FOR COLLISION}

In this section, we divide each message into four 32-bit words and give the representation of internal state differences.

Suppose that a message difference $\Delta m_{t}\left(\Delta m_{t} \neq 0\right)$ is injected at $i$-th encryption (or processing the associated date) step and $\Delta S^{t}=0$, through the differential analysis, it's easy to get internal state differences in $t+1$-th step:

$$
\begin{aligned}
\Delta S_{0}^{t+1} & =0 \\
\Delta S_{1}^{t+1} & =\operatorname{Rotl}\left(\Delta m_{t}, 31\right)<<<64 \\
\Delta S_{2}^{t+1} & =\operatorname{Rotl}\left(\Delta m_{t}, 7\right)<<<32 \\
\Delta S_{3}^{t+1} & =\operatorname{Rotl}\left(\operatorname{Rotl}\left(\Delta m_{t}, 31\right) \oplus \Delta m_{t}, 22\right)<<<32 \\
\Delta S_{4}^{t+1}= & \operatorname{Rotl}\left(S_{0}^{t+1} \& \Delta S_{1}^{t+1} \oplus\left(\Delta S_{2}^{t+1}>>>32\right) \oplus \Delta m_{t}, 13\right) \\
& <<<64
\end{aligned}
$$

Note: For convenience, we perform the left rotation for $\Delta S_{3}^{t+1}$ and $\Delta S_{4}^{t+1}$ in advance.

If and only if $\Delta S_{i}^{t+2}=0(0 \leq i \leq 4)$, the internal state collision occurs. Because we only concern that whether the difference is zero and external operation $\operatorname{Rotl}(x, n)$ doesn't influence the results, we leave out $\operatorname{Rotl}(x, n)$. Each element of state differences after the second step is shown as:

$$
\begin{aligned}
\Delta S_{0}^{t+2}= & \left(S_{1}^{t+1} \& \Delta S_{2}^{t+1}\right) \oplus\left(\Delta S_{1}^{t+1} \& S_{2}^{t+1}\right) \oplus \\
& \left(\Delta S_{1}^{t+1} \& \Delta S_{2}^{t+1}\right) \oplus\left(\Delta S_{3}^{t+1}>>>32\right) ; \\
\Delta S_{1}^{t+2}= & \Delta S_{1}^{t+1} \oplus\left(S_{2}^{t+1} \& \Delta S_{3}^{t+1}\right) \oplus\left(\Delta S_{2}^{t+1} \& S_{3}^{t+1}\right) \oplus \\
& \left(\Delta S_{2}^{t+1} \& \Delta S_{3}^{t+1}\right) \oplus\left(\Delta S_{4}^{t+1}>>>64\right) \oplus \Delta m_{t+1} ; \\
\Delta S_{2}^{t+2}= & \Delta S_{2}^{t+1} \oplus\left(S_{3}^{t+1} \& \Delta S_{4}^{t+1}\right) \oplus\left(\Delta S_{3}^{t+1} \& S_{4}^{t+1}\right) \oplus \\
& \left(\Delta S_{3}^{t+1} \& \Delta S_{4}^{t+1}\right) \oplus \Delta m_{t+1} ; \\
\Delta S_{3}^{t+2}= & \Delta S_{3}^{t+1} \oplus\left(\Delta S_{4}^{t+1} \& S_{0}^{t+2}\right) \oplus \Delta m_{t+1} ; \\
\Delta S_{4}^{t+2}= & \Delta S_{4}^{t+1} \oplus \Delta m_{t+1} .
\end{aligned}
$$

It should be noted that $\Delta S^{t+2}=0$ is a precondition, then we analyze the differences after the second step.

Now we discuss the difference distribution in the following situations with different values of $\mathrm{WB}\left(\Delta m_{t}\right)$. Remember that $\alpha_{i}(1 \leq i \leq 4)$ represents a 32-bit word difference.

\section{A. When $\operatorname{WB}\left(\Delta m_{t}\right)=1$}

Now, we assume that there is only one nonzero word difference in $\Delta m_{t}, \Delta m_{t}=\left(\alpha_{1}, 0,0,0\right), \alpha_{1} \neq 0$.

$$
\begin{aligned}
\Delta S_{1}^{t+1} & =0 \alpha^{\prime} 00, \text { where } \alpha^{\prime}=\alpha<<31 ; \\
\Delta S_{2}^{t+1} & =00 \alpha^{\prime \prime} 0, \text { where } \alpha^{\prime \prime}=\alpha<<7, i=1,2 ;
\end{aligned}
$$


$\Delta S_{3}^{t+1}=00 \beta 0$, where $\beta=\left(\alpha \oplus \alpha^{\prime}\right)<<<22$;

$\Delta S_{4}^{t+1}=0 \gamma 0 u$, where $\gamma=\left(\alpha \oplus \alpha^{\prime}\right)<<<13$, $u$ is unknown difference.

Because of $\Delta S_{4}^{t+2}=\Delta S_{4}^{t+1} \oplus \Delta m_{t+1}$ and $\Delta S_{4}^{t+2}=0$, we get $\Delta m_{t+1}=\Delta S_{4}^{t+1}=0 \gamma 0 u$. $\Delta S^{t+2}$.

Through update function, we know the presentation of

$$
\begin{aligned}
\Delta S_{0}^{t+2}= & S_{1}^{t+1} \& \Delta S_{2}^{t+1} \oplus \Delta S_{1}^{t+1} \& S_{2}^{t+1} \oplus \Delta S_{1}^{t+1} \& \Delta S_{2}^{t+1} \\
& \oplus \Delta S_{3}^{t+1} \gg \gg>32 \\
= & 0 u u 0 \oplus 000 \beta=0 u и \beta
\end{aligned}
$$

To ensure $\Delta S_{0}^{t+2}=0$, we deduce $\beta=0$, namely $\alpha \oplus \alpha<<<31=0$, then $\alpha=1^{32}$ must be established. Therefore we can deduce $\alpha^{\prime}=\alpha^{\prime \prime}=1^{32}$ and $\beta=\gamma=0$, furthermore $\Delta S_{3}^{t+2}=0000, \Delta S_{4}^{t+2}=\Delta m_{t+1}=000 u$.

Supposed $\Delta S_{1}^{t+2}=0$, we can get

$$
\begin{aligned}
\Delta S_{2}^{t+2} & =\Delta S_{2}^{t+1} \oplus S_{3}^{t+1} \& \Delta S_{4}^{t+1} \oplus \Delta m_{t+1} \\
& =00 \alpha^{\prime \prime} 0 \oplus 000 u \oplus 000 u=00 \alpha^{\prime \prime} u \neq 0
\end{aligned}
$$

It contradicts to $\Delta S_{0}^{t+2}=0$, The above results also hold for $\Delta m_{t}=0 \alpha_{1} 00 \quad, \quad \Delta m_{t}=00 \alpha_{1} 0$ and $\Delta m_{t}=000 \alpha_{1} \quad$, so $\mathrm{WB}\left(\Delta m_{t}\right) \neq 1$.

\section{B. When $\operatorname{WB}\left(\Delta m_{t}\right)=2$}

There are two cases:

Case $1 \Delta m_{t}=00 \alpha_{1} \alpha_{2}\left(\alpha_{i} \neq 0, i=1,2\right)$

$\Delta S_{1}^{t+1}=\alpha_{1}^{\prime} \alpha_{2}^{\prime} 00$, where $\alpha_{i}^{\prime}=\alpha_{i} \ll<31, i=1,2$;

$\Delta S_{2}^{t+1}=0 \alpha_{1}^{\prime \prime} \alpha_{2}^{\prime \prime} 0$, where $\alpha_{i}^{\prime \prime}=\alpha_{i}<<<7, i=1,2$;

$\Delta S_{3}^{t+1}=0 \beta_{1} \beta_{2} 0$, where $\beta_{i}=\left(\alpha_{i} \oplus \alpha_{i}^{\prime}\right)<<<22, i=1,2$;

$\Delta S_{4}^{t+1}=\gamma_{1} \gamma_{2} u u, \gamma_{i}=\left(\alpha_{i} \oplus \alpha_{i}^{\prime}\right)<<<13, i=1,2$. u refers to an unknown difference,

$$
\Delta m_{t+1}=\Delta S_{4}^{t+1}=\gamma_{1} \gamma_{2} u u \text {. }
$$

Now we analyze the $\Delta S_{0}^{t+2}$.

$$
\begin{aligned}
\Delta S_{0}^{t+2}= & S_{1}^{t+1} \& \Delta S_{2}^{t+1} \oplus \Delta S_{1}^{t+1} \& S_{2}^{t+1} \oplus \Delta S_{1}^{t+1} \& \Delta S_{2}^{t+1} \\
& \oplus \Delta S_{3}^{t+1} \gg>>32 \\
= & \text { иии } 0 \oplus 00 \beta_{1} \beta_{2}=и и и \beta_{2}
\end{aligned}
$$

To ensure $\Delta S_{0}^{t+2}=0$, we deduce $\beta_{2}=0$, it's easy to get $\alpha^{\prime}=\alpha^{\prime \prime}=1^{32}, \gamma_{2}=0$. So $\Delta S_{3}^{t+1}=0 \beta_{1} 00, \Delta S_{4}^{t+1}=\Delta m_{t+1}=\gamma_{1} 0 u u$.

Supposed $\Delta S_{1}^{t+2}=\Delta S_{2}^{t+2}=0$, we can obtain

$$
\begin{aligned}
\Delta S_{3}^{t+2} & =\Delta S_{3}^{t+1} \oplus \Delta S_{4}^{t+1} \& S_{0}^{t+2} \oplus \Delta m_{t+1} \\
& =0 \beta_{1} 00 \oplus u 0 u u=u \beta_{1} u u
\end{aligned}
$$

To ensure $\Delta S_{3}^{t+2}=0$, we deduce $\beta_{1}=0, \alpha^{\prime}=\alpha^{\prime \prime}=1^{32}$ and $\gamma_{1}=0$, therefore $\Delta S_{3}^{t+1}=0000, \Delta S_{4}^{t+1}=\Delta m_{t+1}=00 u u$. As for $\Delta S_{2}^{t+2}$, we get that

$$
\begin{aligned}
\Delta S_{2}^{t+2} & =\Delta S_{2}^{t+1} \oplus S_{3}^{t+1} \& \Delta S_{4}^{t+1} \oplus \Delta m_{t+1} \\
& =0 \alpha_{1}^{\prime \prime} \alpha_{2}^{\prime \prime} 0 \oplus 00 u u=0 \alpha_{1}^{\prime \prime} u u \neq 0
\end{aligned}
$$

It contradicts to $\Delta S_{2}^{t+2}=0$. The above results also hold for $\Delta m_{t}=0 \alpha_{1} \alpha_{2} 0, \Delta m_{t}=00 \alpha_{1} \alpha_{2}$ and $\Delta m_{t}=\alpha_{1} 00 \alpha_{2}$.

Case $2 \Delta m_{t}=0 \alpha_{1} 0 \alpha_{2}\left(\alpha_{i} \neq 0, i=1,2\right)$

$\Delta S_{1}^{t+1}=0 \alpha_{2}^{\prime} 0 \alpha_{1}^{\prime}$, where $\alpha_{i}^{\prime}=\alpha_{i} \ll<<31, i=1,2$;

$\Delta S_{2}^{t+1}=\alpha_{1}^{\prime \prime} 0 \alpha_{2}^{\prime \prime} 0$, where $\alpha_{i}^{\prime \prime}=\alpha_{i} \ll<7, i=1,2$;

$\Delta S_{3}^{t+1}=\beta_{1} 0 \beta_{2} 0$, where $\beta_{i}=\left(\alpha_{i} \oplus \alpha_{i}^{\prime}\right)<<22, i=1,2$;

$\Delta S_{4}^{t+1}=0 u 0 u \cdot u$ denotes unknown difference.

$\Delta m_{t+1}=\Delta S_{4}^{t+1}=0 u 0 u$.

Supposed $\Delta S_{0}^{t+2}=\Delta S_{1}^{t+2}=\Delta S_{2}^{t+2}=0$, we analyze $\Delta S_{3}^{t+2}$.

$$
\Delta S_{3}^{t+2}=\Delta S_{3}^{t+1} \oplus \Delta S_{4}^{t+1} \& S_{0}^{t+2} \oplus \Delta m_{t+1}
$$$$
=\beta_{1} 0 \beta_{2} 0 \oplus 0 u 0 u=\beta_{1} u \beta_{2} u
$$

To ensure $\Delta S_{3}^{t+2}$, we get $\beta_{1}=\beta_{2}=0$ and $\gamma_{1}=\gamma_{2}=0$, therefore $\Delta S_{3}^{t+1}=0000$. Take consideration of $\Delta S_{2}^{t+2}$,

$$
\begin{aligned}
\Delta S_{2}^{t+2} & =\Delta S_{2}^{t+1} \oplus S_{3}^{t+1} \& \Delta S_{4}^{t+1} \oplus \Delta m_{t+1}=\alpha_{1}^{\prime \prime} 0 \alpha_{2}^{\prime \prime} 0 \oplus 0 u 0 u \\
& =\alpha_{1}^{\prime \prime} u \alpha_{2}^{\prime \prime} u \neq 0
\end{aligned}
$$

It contradicts to $\Delta S_{2}^{t+2}=0$, The same results also hold for $\Delta m_{t}=0 \alpha_{2} 0 \alpha_{4}$, so $\operatorname{WB}\left(\Delta m_{t}\right) \neq 2$.

C. When $\operatorname{WB}\left(\Delta m_{t}\right)=3$

Assume that $\Delta m_{t}=\left(0, \alpha_{1}, \alpha_{2}, \alpha_{3}\right)\left(\alpha_{i} \neq 0, i=1,2,3\right)$, we can deduce the followings.

$\Delta S_{1}^{t+1}=\alpha_{2}^{\prime} \alpha_{3}^{\prime} 0 \alpha_{1}^{\prime}$, where $\alpha_{i}^{\prime}=\alpha_{i} \ll<<31, i=1,2,3 ;$

$\Delta S_{2}^{t+1}=\alpha_{1}^{\prime \prime} \alpha_{2}^{\prime \prime} \alpha_{3}^{\prime \prime} 0$, where $\alpha_{i}^{\prime \prime}=\alpha_{i} \ll<<7, i=1,2,3$;

$\Delta S_{3}^{t+1}=\beta_{1} \beta_{2} \beta_{3} 0$, where $\beta_{i}=\left(\alpha_{i} \oplus \alpha_{i}^{\prime}\right) \ll<22, i=1,2,3$;

$\Delta S_{4}^{t+1}=\gamma_{1}$ иии $, \gamma_{i}=\left(\alpha_{i} \oplus \alpha_{i}^{\prime}\right)<<<13, i=1,2,3$, $и$ denotes unknown difference.

$$
\Delta m_{t+1}=\Delta S_{4}^{t+2}=\gamma_{1} u и u .
$$

We can obtain the internal state difference $\Delta S^{t+2}$ as follows: 


$$
\begin{gathered}
\Delta S_{0}^{t+2}=S_{1}^{t+1} \& \Delta S_{2}^{t+1} \oplus \Delta S_{1}^{t+1} \& S_{2}^{t+1} \oplus \Delta S_{1}^{t+1} \& \Delta S_{2}^{t+1} \\
\quad \Delta S_{3}^{t+1} \gg>>\text { ииии } \\
\Delta S_{1}^{t+2}=\Delta S_{1}^{t+1} \oplus S_{2}^{t+1} \& \Delta S_{3}^{t+1} \oplus \Delta S_{2}^{t+1} \& S_{3}^{t+1} \oplus \Delta S_{2}^{t+1} \& \Delta S_{3}^{t+1} \\
\quad \oplus \Delta S_{4}^{t+1} \gg \gg>64 \oplus \Delta m_{t+1}=\text { ииии } \\
\Delta S_{2}^{t+2}=\Delta S_{2}^{t+1} \oplus S_{3}^{t+1} \& \Delta S_{4}^{t+1} \oplus \Delta S_{3}^{t+1} \& S_{4}^{t+1} \oplus \Delta S_{3}^{t+1} \& \Delta S_{4}^{t+1} \\
\oplus \Delta m_{t+1}=\text { ииии } \\
\Delta S_{3}^{t+2}=\Delta S_{3}^{t+1} \oplus \Delta S_{4}^{t+1} \& S_{0}^{t+2} \oplus \Delta m_{t+1}=\text { ииии }
\end{gathered}
$$

Each 128-bit element of $\Delta S^{t+2}$ could be 0 , therefore $\mathrm{WB}\left(\Delta m_{t}\right)=3$ is possible.

\section{When $\operatorname{WB}\left(\Delta m_{t}\right)=4$}

We know $\Delta m_{t}=\left(\alpha_{1}, \alpha_{2}, \alpha_{3}, \alpha_{4}\right) \quad\left(\alpha_{i} \neq 0, i=1,2,3,4\right)$. After one step update, each 32-bit word of $\Delta S_{1}^{t+1}$ and $\Delta S_{2}^{t+1}$ are non-zero, meanwhile, each 32-bit word of $\Delta S_{3}^{t+1}$ and $\Delta S_{4}^{t+1}$ is uncertain, through our analysis, each 128-bit element of $\Delta S^{t+2}$ could be 0 ,therefore $\operatorname{WB}\left(\Delta m_{t}\right)=4$ is possible.
We can draw the conclusion below.

Conclusion 1. For MORUS, if the message difference $\Delta m_{t}\left(\Delta m_{t} \neq 0\right)$ is injected at $i$-th encryption (or processing the associated date) step and gets eliminated at the next step, then $\operatorname{WB}\left(\Delta m_{t}\right) \geq 3$ must be true.

\section{The LOWER BOUND OF DifFERENCE WeIgHT FOR COLLISION}

Because the collision probability is related to the weight of message difference, we discuss the bit-oriented necessary conditions in this section. Through theory deduction, we complete exhaustion when the weight of message difference is less than or equal to 5 .

In the end the experiment results indicate the following conclusion.

Conclusion 2. For MORUS, when the weight of message difference is less than or equal to 5 , there is no collision.

Table I. compares the result of this paper with that of designers on collision cryptanalysis.

TABLE I. RESULTS COMPARISON

\begin{tabular}{|c|c|c|c|c|}
\hline Analyst & Analysis method & Idea & Difference distribution & Weight of difference \\
\hline Designers & Experiment & - & - & $\mathrm{W}\left(\Delta m_{t}\right) \geq 26$ \\
\hline This paper & Theory deduction & Partition method & $\mathrm{WB}\left(\Delta m_{t}\right) \geq 3$ & $\mathrm{~W}\left(\Delta m_{t}\right) \geq 5$ \\
\hline
\end{tabular}

\section{CONCLUSIONS}

In this work, we focus on the security of message authentication in MORUS. Through partition method, we found out the distribution of input difference is determined, namely $\mathrm{WB}\left(\Delta m_{t}\right) \geq 3$. Finally, by experiments, we deduce the lower bound of difference is 5 .

The new idea we used to find the distribution of wordoriented difference for collision is universal, which can be applied to other authenticated ciphers. Moreover, we will evaluate the ability of MORUS in resisting other attacks.

\section{ACKNOWLEDGMENT}

This work was supported by the National Natural Science Foundation of China (Grant No. 61572516, 61272041 and 61272488).

\section{REFERENCES}

[1] M. Bellare et al., "Authenticated encryption: Relations among notions and analysis of the generic composition paradigm," Journal of Cryptology, vol. 21, no. 4, Jan. 2008, pp. 469-491.

[2] K. Hickman et al., "The SSL protocol," Netscape Communications Corp, 1995, 501.

[3] D. J. Bernstein, "Caesar: Competition for authenticated encryption: Security, applicability, and robustness," 2015.
[4] J. Daemen et al., "AES the advanced encryption standard," The Design of Rijndael, 2002.

[5] ECRYPT, European Network of Excellence in Cryptology: "The Stream Cipher Project: eSTREAM,” IST-2002-507932. http://www.ecrypt.eu.org/stream.

[6] C. Dobrauning et al., "Heuristic Tool for Linear Cryptanalysis with Applications to CAESAR Candidates," ASIACRYPT, Auckland, New Zealand, 2015, pp.490-509.

[7] T. Peyrin et al., "Cryptanalysis of JAMBU," FSE 2015, Istanbul, Turkey, Mar. 2015, pp. 264-281.

[8] M. Salam et al., "Investigating Cube Attack on the Authenticated Encryption Stream Cipher ACORN," ATIS 2016, Cairns, QLD, Australia, Oct. 2016, pp. 15-26

[9] H. Wu et al., "The authenticated cipher MORUS (v1)," CAESAR submissions. http://competitions.cr.yp.to/ round2/morusv11.pdf, 2015.

[10] A. Mileva et al., "Analysis of the Authenticated Cipher MORUS (v1)," Int. Conf. BalkanCryptSec 2015, Koper, Slovenia, Sept. 2015, pp. 45-59.

[11] P. Zhang et al., "Research on the confusion and diffusion properties of the initialization of MORUS," J. Cryptologic Research, 2015, vol. 2, no. 6, pp. 536-548.

[12] T. Fuhr et al., "Collision Attacks Against CAESAR Candidates Forgery and Key-Recovery Against AEZ and Marble," Advances in Cryptology-ASIACRYPT 2015, Auckland, New Zealand, Nov. 2015, pp. 510-532.

[13] T. Peyrin, "Collision Attack on Grindahl," J. Cryptology, vol. 28, no. 4, January 2015, pp. 879-898. 